(C) 2015 IEEE. Personal use of this material is permitted. Permission from IEEE must be obtained for all other uses, in any current or future media, including reprinting/republishing this material for advertising or promotional purposes, creating new collective works, for resale or redistribution to servers or lists, or reuse of any copyrighted component of this work in other works. 


\section{Extended Finite Element Method for Electromagnetic Fields}

\author{
$\mathrm{Na} \mathrm{Na}$ Duan ${ }^{1}$, Wei Jie $\mathrm{Xu}^{1,2}$, Shu Hong Wang ${ }^{1}$ \\ ${ }^{1}$ State Key Laboratory of Electrical Insulation and Power \\ Equipment, School of Electrical Engineering, \\ Xi'an Jiaotong University, \\ Xi'an 710049, China \\ shwang@mail.xjtu.edu.cn
}

\author{
Jian Guo Zhu², You Guang Guo ${ }^{2}$ \\ ${ }^{2}$ School of Electrical, Mechanical and Mechatronic Systems, \\ University of Technology, Sydney, \\ NSW 2007, Australia \\ jianguo.zhu@uts.edu.au
}

$$
u^{h}(\boldsymbol{X})=\sum_{i \in I} N_{i}(\boldsymbol{X}) u_{i}+\sum_{i \in I^{*}} N_{i}^{*}(\boldsymbol{X}) \cdot[\psi(\boldsymbol{X})] a_{i}
$$

extended finite element method (XFEM) for electromagnetic field locally non-smooth features within finite elements, such as singularities, discontinuities, and high derivatives. An alternative enrichment function is introduced to improve the approximation space of the conventional finite element method (CFEM) such that non-smooth solutions are modeled independent of the mesh. The level set method is employed to describe the interfaces among different materials. To demonstrate the advantages, the XFEM is compared with CFEM by solving a 1D electrical field problem.

Keywords-Extended finite element method; enrichment function; level set; electromagnetic field

\section{INTRODUCTION}

A large number of applications can be seen that the quantities of electromagnetic field change rapidly over length scales which are small in comparison to the solution domain. Such examples include the exponential current distribution due to the skin-effect in a solid conductor subject to a high frequency magnetic field [1], and the current and diamagnetic characteristics in thin superconducting tapes [2], etc.

In past decades, the extended finite element method (XFEM), which was firstly proposed by Belytschko [3]. Aiming to the approximation of non-smooth solutions, the traditional approach is to employ the polynomial approximation, which depends on meshes that conform to discontinuities and are refined near singularities and high gradients [4]. However, in XFEM, the strategy is to enrich a polynomial approximation space such that the non-smooth solutions can be modeled independent of the mesh.

\section{PRINCIPLE OF EXTENDED FINITE ELEMENT METHOD}

The level set is to represent design domains and the material boundaries by continuum level set function. By solving the level set function, interface position is obtained.

In the discretized domain, $I$ is the set of all nodes, $I^{*}$ is the set of the enriched nodes, $I^{*} \in I$.

The approximation of a potential function $u(\boldsymbol{X})$ may be defined as [3] where $\mathrm{Ni}$ and $\mathrm{N}_{i}^{*}$ are conventional finite element method (CFEM) shape functions, in general, $N i=N_{i}{ }^{*}$. The coefficients $u_{i}$ belong to the CFEM part and $a_{i}$ are additional nodal unknowns. The function $\psi(\boldsymbol{X})$ is called an enrichment function. The products $N i(\boldsymbol{X}) \cdot \psi(\boldsymbol{X})$ are local enrichment functions.

The enrichment function for weak discontinuity may be chosen as the absolute value of the zero level set function.

$$
\psi\left(\boldsymbol{X}-\boldsymbol{X}^{*}\right)=\operatorname{abs}\left(\phi\left(\boldsymbol{X}-\boldsymbol{X}^{*}\right)\right)=\left|\phi\left(\boldsymbol{X}-\boldsymbol{X}^{*}\right)\right|
$$

where $\phi$ is the level set function, and $X^{*}$ the closest point on the interface to the point $\boldsymbol{X}$.

\section{SimUlation VERIFICATION OF XFEM}

In order to apply the XFEM to electromagnetic field analysis, the electric field distributions between parallel plate electrodes, which contain two and three dielectrics, are calculated by using XFEM, respectively.

\section{A. Two Dielectrics}

Fig.1 shows the parallel plate electrode system. In Fig.1, the distances $d_{1}$ and $d_{2}$ are all $9 \mathrm{~m}$, the permittivities of two dielectrics $\varepsilon_{1}=9 \varepsilon_{0}$ and $\varepsilon_{2}=3 \varepsilon_{0}$, respectively. The imposed voltage $U_{0}$ is $10 \mathrm{~V}$.

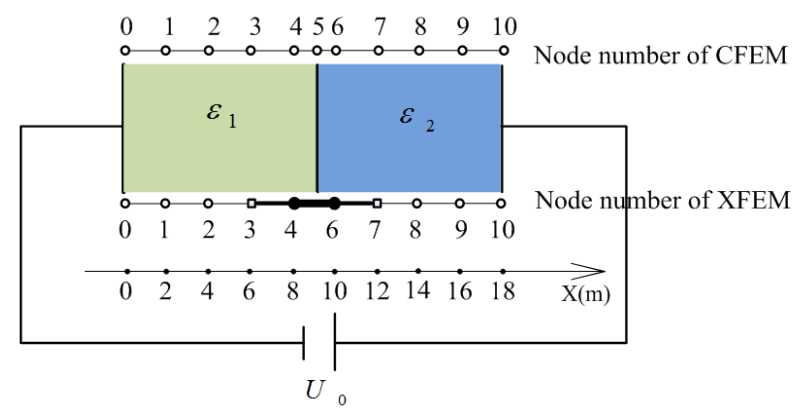

Fig.1. The parallel plate electrode system containing two dielectrics 
The nodes are numbered as shown in Fig.1. The element is denoted as "Element $i-j$ ", in which $i$ and $j$ are the numbers of left and right nodes in one element. The Node 5 is used in classical FE and ignored in XFEM analysis. The Element 4-6 is the enriched element in which the interface passes through. Elements 3-4 and 6-7 are blending elements.

The distance function, i.e., the absolute value of level set function $|\psi(x)|=|x-9|$, is chosen as the enrichment function. The electrical potential approximation may be written as

$$
u^{h}(x)=\sum_{i \in I} N_{i}(x) u_{i}+\sum_{i \in I^{*}} N_{i}^{*}(x) \cdot|(x-9)| a_{i} .
$$

where shape functions $N i$ and $N_{i}{ }^{*}$ are defined as the same linear interpolation.

Fig. 2 shows the electrical potential distribution calculated by XFEM and CFEM.

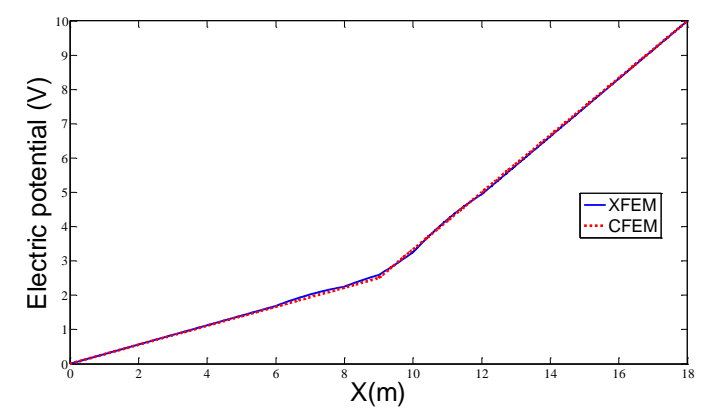

Fig.2. The electrical potential distribution calculated by XFEM and CFEM.(Two dielectrics)

According to Fig.2, the results calculated by using XFEM are closely agreed with those by using CEFM.

\section{B. Three Dielectrics with Thin Layer}

Fig.3 shows another parallel plate electrode system containing three dielectrics. The middle of the three dielectrics may be regarded as a thin layer material because the width of middle dielectric is $6 \%$ of that of the others. The permittivities of three dielectrics are $\varepsilon_{1}=2 \varepsilon_{0}, \varepsilon_{2}=4 \varepsilon_{0}$ and $\varepsilon_{1}=3 \varepsilon_{0}$, respectively. The imposed voltage $U_{0}$ is $10 \mathrm{~V}$.

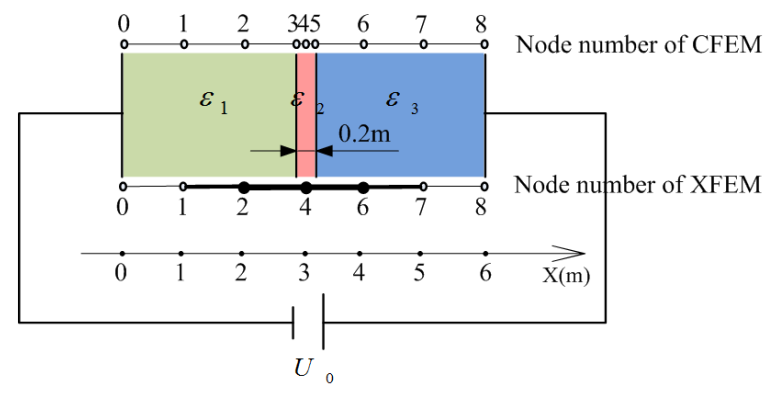

Fig.3. The parallel plate electrode system containing three dielectrics

The related error comparison between CFEM and XFEM is listed in Table I.
TABLE I. THE COMPARISON OF NODAL ElECTRICAL POTENTIAL

\begin{tabular}{|l|l|c|c|}
\hline \multirow{2}{*}{$\begin{array}{c}\text { No. of } \\
\text { Node }\end{array}$} & \multicolumn{3}{|c|}{ Simulation Results } \\
\cline { 2 - 4 } & $\mathbf{X F E M} \boldsymbol{U}_{\boldsymbol{i}}(\mathbf{V})$ & $\mathbf{C F E M} \boldsymbol{U}_{\boldsymbol{i}}(\mathbf{V})$ & $\begin{array}{c}\text { Related Error } \\
(\%)\end{array}$ \\
\hline 0 & 0 & 0 & 0.00 \\
\hline 1 & 1.1662 & 1.1364 & 2.62 \\
\hline 2 & 2.3322 & 2.2727 & 2.62 \\
\hline 3 & 3.38532 & 3.3523 & 0.98 \\
\hline 4 & 5.33556 & 5.4546 & -2.18 \\
\hline 5 & 7.6678 & 7.7273 & -0.77 \\
\hline 6 & 10 & 10 & 0.00 \\
\hline
\end{tabular}

The maximum of related errors is less than $3 \%$ and the results calculated by using XFEM are acceptable from the viewpoint of engineering. The accuracy of electrical potentials in blending elements may be less than that in enriched element. It is suggested that the enrichment function should be modified for improvement of the performance of XFEM.

\section{CONCLUSIONS}

In this paper, the XFEM for electromagnetic field is presented. The enrichment function, described by using the level set function and is associated with the interfaces, may improve the standard finite element approximation in the cases of discontinuities, singularities, high derivatives, or other nonsmooth properties. One of the advantages of XFEM is that the interfaces do not align with the edges of meshes, because the enrichment function can deal with the interfaces. The mesh refinement technique may not be used for thin layer problem. The numerical simulation, based on one dimensional node elements to solve the Maxwell equations of electric field in a domain containing multi-materials as well thin layer dielectric, concluded that XFEM is able to simulate discontinuities and thin layer behavior of the electric field on a mesh that is independent from the interface location.

\section{ACKNOWLEDGMENT}

This work is supported in part by the National Natural Science Foundation of China (NSFC) (Grant No. 51177116).

\section{REFERENCES}

[1] J. Zhang, S. H. Wang, J. Qiu, H. B. Li, Q. H. Zhang, J. G. Zhu, Y. G. Guo, "Finite element analysis and evaluation of stator insulation in high voltage synchronous motor", IEEE Transactions on Magnetics, vol. 48, no. 2, 2012, pp. 955-958.

[2] X. Y. Liu, S. H. Wang, J. Qiu, J. G. Zhu, Y. G. Guo, Z. W. Lin, "Robust optimization in HTS cable based on design for six sigma," IEEE Transactions on Magnetics, vol. 44, no. 6, 2008, pp. 978 - 981 .

[3] T. P Fries, T. Belytschko, "The extended/generalized finite element method: an overview of the method and its applications," International journal for numerical methods in engineering, vol. 84. no. 3, 2010, pp. 253-304, 2010.

[4] O. C. Zienkiewicz, R. L. Taylor. The Finite Element Method, vol. 1-3. Butterworth-Heinemann: Oxford, 2000. 
